\title{
Transient visual loss and delayed resolution of vitreomacular traction after intravitreal ocriplasmin
}

This article was published in the following Dove Press journal:

Drug, Healthcare and Patient Safety

25 November 2014

Number of times this article has been viewed

\author{
Joanna DaCosta ${ }^{1,2}$ \\ Saad Younis' \\ 'The Western Eye Hospital, Imperial \\ College Healthcare National Health \\ Service Trust, London, United Kingdom; \\ ${ }^{2}$ Barts Health National Health Service \\ Trust, St Bartholomew's Hospital, West \\ Smithfield, London, United Kingdom
}

Correspondence: Joanna DaCosta Ocular Oncology Service, Barts Health National Health Service Trust, St Bartholomew's Hospital, West Smithfield, London ECIA 7BE, United Kingdom

Tel +44203465 6863

Fax +44203465 5936

Email dacostajoanna@hotmail.co.uk

\begin{abstract}
Vitreomacular traction and vitreomacular traction with macular hole are conditions associated with visual disturbance and decreased visual acuity. Recent studies have demonstrated that ocriplasmin is effective and safe in specific patient groups with vitreomacular traction, and hence is recommended as a treatment option. We present here a case report of a patient treated with intravitreal ocriplasmin associated with severe loss of vision and delayed resolution of vitreomacular traction. This report highlights that it is important for patients to be made fully aware of the risks of intravitreal ocriplasmin and the prognosis for visual improvement.
\end{abstract}

Keywords: ocriplasmin, macular hole, visual loss, vitreoretinal

\section{Case report}

A 97-year-old woman developed decreased vision in her left eye over a period of 4 weeks. Past ocular history included uneventful bilateral cataract surgery and bilateral early dry age-related macular degeneration (AMD). Vision was 6/30 on the right and 6/38 LogMAR on the left. Spectral domain optical coherence tomography (SD-OCT; Cirrus, Carl Zeiss Meditec, Dublin, CA, USA) of the left eye showed vitreomacular traction with a small stage 2 macular hole ${ }^{1,2}$ and no epiretinal membrane (Figure 1). The patient was informed that ocriplasmin had been shown to statistically significantly resolve vitreomacular adhesion when compared with placebo in clinical trials, and the option of vitrectomy was discussed. Fully informed consent was obtained, and the risk for complications with intravitreal injections such as loss of vision, retinal detachment, lens damage, and endophthalmitis was documented on the consent form. The patient was made aware that there would be a guarded prognosis for visual improvement. She proceeded to receive treatment with intravitreal ocriplasmin $0.125 \mathrm{mg}(0.1 \mathrm{~mL})$ to her left eye.

One day after treatment, vision had reduced to hand movements, with symptoms of blue and dark spots in the left eye. Ocular examination revealed normal intraocular pressure and no sign of endophthalmitis, retinal detachment, or hemorrhage. No other cause of reduced vision was identified. Repeat SD-OCT revealed subretinal fluid with vitreomacular traction (Figure 2). There is a small irregular area of retinal pigment epithelium elevation (Figure 2). The patient declined fluorescein and indocyanine green angiography, and clinically, this appeared to be related to dry age-related macular changes. There appeared to be attenuation and discontinuation of the inner segment/ outer segment, ellipsoid layer.

Twenty-eight days after treatment, the patient's vision had improved to 6/48 LogMAR (Figure 3), and the subretinal fluid had resolved with persistent vitreomacular traction. 


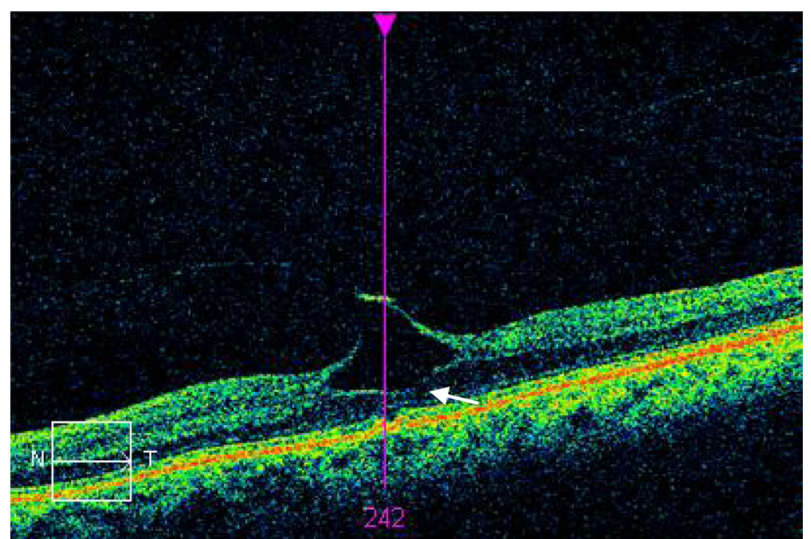

Figure I Vitreomacular traction with a small stage 2 macular hole (arrow).

The patient elected not to proceed with pars plana vitrectomy.

At 56 days after treatment, the vitreomacular traction had resolved with sustained improvement of vision (Figure 4). This appeared to be associated with improved continuity of the inner segment/outer segment (ellipsoid layer).

\section{Discussion}

Our case report shows that ocriplasmin caused severe visual loss postinjection associated with subretinal fluid, and that vitreomacular traction may resolve up to 2 months after ocriplasmin injection.

Normal aging is accompanied by physiological changes in the vitreous gel of the eye. After the age of 40 years, the vitreous undergoes progressive liquefaction (synchysis). ${ }^{3}$ Typically, by the age of 80 years, about $50 \%$ of the vitreous gel has been liquefied, resulting in weakened adhesion between the vitreous and retina. ${ }^{3}$ Posterior vitreous detachment is more common in postmenopausal women than men; this is postulated to be a result of the effects of decreased

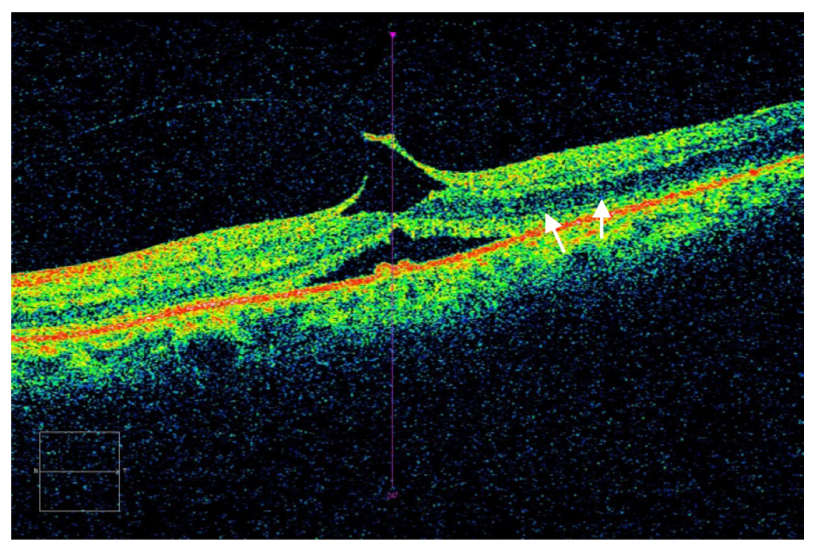

Figure 2 Persistent vitreomacular traction with subretinal fluid; arrows show attenuation and discontinuation of the ellipsoid layer.

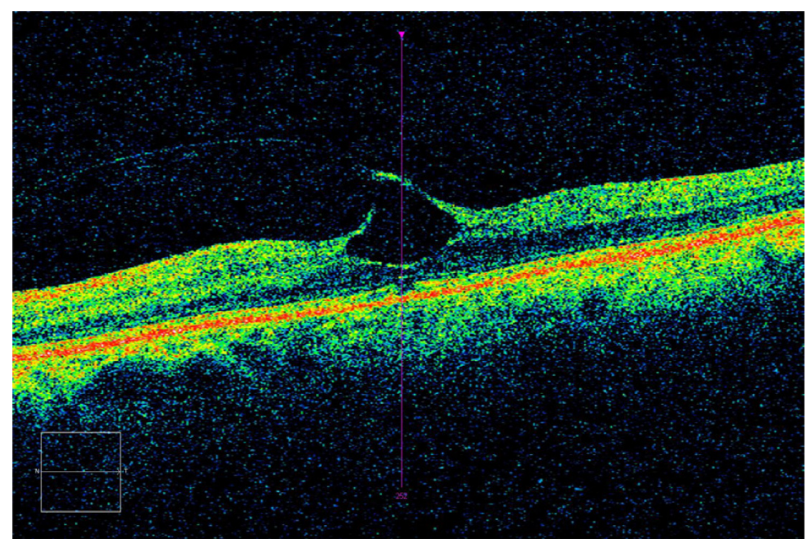

Figure 3 Persistent vitreomacular traction with resolved subretinal fluid.

estrogen on connective tissue, such as that present within the vitreous gel. ${ }^{4}$

The patient in our case report was an elderly woman, aged 97 years, with early dry AMD. Clinically, AMD appears to primarily involve the retinal pigment epithelium and outer retinal layers. However, evidence suggests that the vitreous may contribute to the pathogenesis and progression of AMD. For example, Mojana et $\mathrm{al}^{5}$ conducted a retrospective study with SD-OCT to compare vitreoretinal adhesions in three groups: eyes with wet AMD, eyes with dry AMD, and control eyes. Eyes with wet AMD were significantly more likely to show evidence of vitreomacular adhesion. The patient described in our case report was elderly and female, which would suggest weakened vitreomacular adhesion; however, in the context of early AMD, this may have contributed to increased vitreomacular adhesion.

Results of clinical trials ${ }^{6}$ and the package insert of ocriplasmin $^{7}$ (Jetrea $^{\circledR}$, ThromboGenics, Leuven, Belgium) report transient blindness, photopsia, dyschromatopsia, and electroretinogram (ERG) changes occurring more frequently

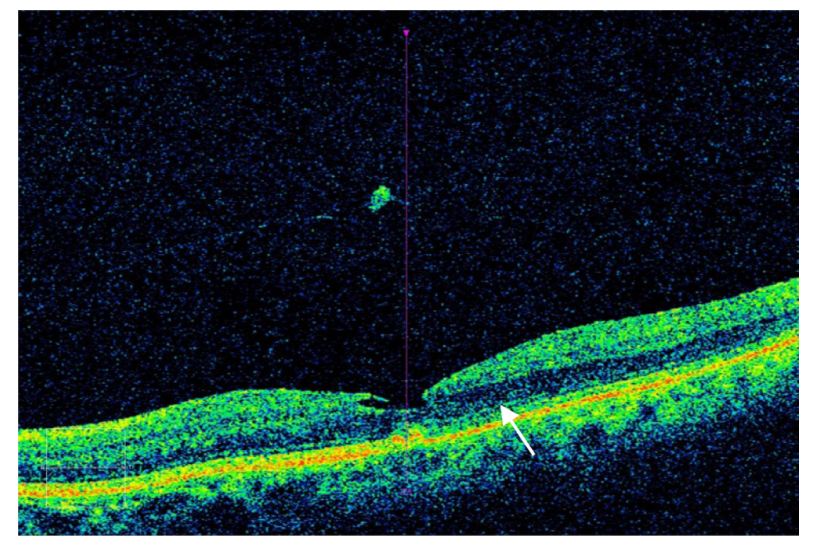

Figure 4 Resolution of vitreomacular traction, with the arrow showing improved continuity of the ellipsoid layer. 
in those patients treated compared with in placebo-treated patients.

Freund et $\mathrm{al}^{8}$ described a 67-year-old woman with a macular hole associated with vitreomacular adhesion and epiretinal membrane who developed transient loss of vision 1 week after intravitreal ocriplasmin. Eye-tracked SD-OCT demonstrated resolution of vitreomacular adhesion with disruption of the ellipsoid zone, indicating damaged photoreceptor outer segments. Despite the persistence of the macular hole, the patient's vision recovered 3 weeks later. This was associated with improvement of the integrity of the ellipsoid zone, which is suggestive of partial recovery of photoreceptor outer segment function. It was proposed that ocriplasmin exerts a disruptive effect on the photoreceptor outer segments, which appears to be partially reversible. ${ }^{8}$ Singh et $\mathrm{al}^{9}$ was the first study to quantify the extent of outer retinal changes seen in patients receiving ocriplasmin. Seven of 17 patients experienced transient outer segment ellipsoid zone loss on SD-OCT, which resolved after an average period of 29.3 days. This was also correlated with subretinal fluid, which resolved after an average period of 30 days.

Recent case reports such as Tibbetts et $\mathrm{al}^{10}$ and Fahim et $\mathrm{al}^{11}$ have described a more diffuse effect of ocriplasmin on the photoreceptors. Tibbetts et $\mathrm{al}^{10}$ described a 71 -year-old woman with symptomatic vitreomacular traction who experienced persistent darkening of vision for 4 months after receiving intravitreal ocriplasmin. The symptoms of darkened vision corresponded with disruption of the photoreceptor inner segment-outer segment (ellipsoid) layer and reduce a- and b-wave ERG amplitudes. However, this was not related to persistent vitreomacular traction, as there was an immediate release of vitreomacular traction.

Full-field ERG revealed a greater reduction in the amplitude of the scotopic compared with the photopic ERG, suggesting rod photoreceptors may be more susceptible to the effects of ocriplasmin. These effects may only occur in patients who are susceptible to prolonged reduction in photoreceptor activity. It was postulated that a diffuse protease enzymatic effect of ocriplasmin throughout the retina may account for the visual darkening adverse effects of ocriplasmin.

Fahim et $\mathrm{al}^{11}$ reported a case of persistent visual loss associated with acute severe panretinal dysfunction after ocriplasmin injection for a small macular hole with vitreomacular adhesion. This was associated with Goldmann visual field constriction, anisocoria, attenuated retinal vessels, disruption of outer retinal signals on SD-OCT, and severely reduced ERG responses. Fahim et $\mathrm{al}^{11}$ postulated that possible mechanisms of retinal injury from ocriplasmin injection included either enzymatic activity of ocriplasmin on the retinal extracellular matrix or other toxic effects not specific to the proteinase activity of ocriplasmin.

The enzymatic effects of ocriplasmin on the cleavage of laminin, a component of the photoreceptor-bipolar synapse, were consistent with ERG findings of reduced b-waves compared with a-waves. This suggests postreceptor (eg, bipolar cell) cell dysfunction in addition to decreased photoreceptor activity.

The results of clinical trials ${ }^{6}$ were statistically significant, showing that vitreomacular adhesion resolved in $26.5 \%$ of ocriplasmin-injected eyes compared with in $10.1 \%$ of placeboinjected eyes. The primary end point of the study ${ }^{6}$ was resolution of vitreomacular adhesion at 28 days, determined by OCT evaluation. Visual improvements with ocriplasmin were less encouraging, demonstrating that anatomical resolution of vitreomacular traction noted with OCT is not a surrogate marker for functional visual improvement. ${ }^{6}$

The National Institute of Health and Care Excellence has recommended ocriplasmin as a treatment option for patients with vitreomacular traction if no epiretinal membrane is present and a stage 2 macular hole is present (with a diameter of $\leq 400 \mu \mathrm{m}$ ) and/or the patient has severe symptoms. ${ }^{12}$ After our experience with this patient, we have sought to make the consent process for intravitreal ocriplasmin more explicit and to explain to patients that there appear to be specific risks for severe visual loss associated with this drug, in addition to the well-described risks of the intravitreal injection process.

It is important that patients are made fully aware of the risks of ocriplasmin and the prognosis for visual improvement.

\section{Disclosure}

The authors report no conflicts of interest in this work.

\section{References}

1. Gass JD. Idiopathic senile macular hole. Its early stages and pathogenesis. Arch Ophthalmol. 1988;106(5):629-639.

2. Gass JD. Reappraisal of biomicroscopic classification of stages of development of a macular hole. Am J Ophthalmol. 1995;119(6):752-759.

3. Le Goff MM, Bishop PN. Adult vitreous structure and postnatal changes. Eye (Lond). 2008;22(10):1214-1222.

4. Chuo JY, Lee TYY, Hollands H, et al. Risk factors for posterior vitreous detachment: a case-control study. Am J Ophthalmol. 2006;142(6): 931-937.

5. Mojana F, Cheng L, Bartsch DU, et al. The role of abnormal vitreomacular adhesion in age-related macular degeneration: spectral optical coherence tomography and surgical results. Am J Ophthalmol. 2008;146(2):218-227.

6. Stalmans P, Benz MS, Gandorfer A, et al; MIVI-TRUST Study Group Enzymatic vitreolysis with ocriplasmin for vitreomacular traction and macular holes. N Engl J Med. 2012;367(7):606-615. 
7. Jetrea ${ }^{\circledR}$ (ocriplasmin) [package insert]. Iselin, NJ: ThromboGenics; 2013.

8. Freund KB, Shah SA, Shah VP. Correlation of transient vision loss with outer retinal disruption following intravitreal ocriplasmin. Eye (Lond). 2013;27(6):773-774.

9. Singh RP, Li A, Bedi R, et al. Anatomical and visual outcomes following ocriplasmin treatment for symptomatic vitreomacular traction syndrome. Br J Ophthalmol. 2014;98(3):356-360.

10. Tibbetts MD, Reichel E, Witkin AJ. Vision loss after intravitreal ocriplasmin: correlation of spectral-domain optical coherence tomography and electroretinography. JAMA Ophthalmol. 2014;132(4):487-490.
11. Fahim AT, Khan NW, Johnson MW. Acute panretinal structural and functional abnormalities after intravitreous ocriplasmin injection. JAMA Ophthalmol. 2014;132(4):484-486.

12. National Institute for Health and Care Excellence. Ocriplasmin for Treating Vitreomacular Traction. NICE technology appraisal guidance 297. London, UK: National Institute for Health and Care Excellence; October 2013. Available from: http://www.nice.org.uk/guidance/ ta297/documents/vitreomacular-traction-ocriplasmin-final-appraisaldetermination-document2. Accessed October 1, 2014.

\section{Publish your work in this journal}

Drug, Healthcare and Patient Safety is an international, peer-reviewed open-access journal exploring patient safety issues in the healthcare continuum from diagnostic and screening interventions through to treatment, drug therapy and surgery. The journal is characterized by the rapid reporting of reviews, original research, clinical, epidemiological and post-marketing surveillance studies, risk management, health literacy and educational programs across all areas of healthcare delivery. The manuscript management system is completely online and includes a very quick and fair peer-review system. Visit http://www.dovepress.com/ testimonials.php to read real quotes from published authors. 